\title{
Consideraciones Jurídicas en torno a la Ovodonación
}

\author{
Legal issues of egg donation
}

Ronald Cárdenas Krenz

\section{Resumen}

El artículo aborda el discutido tema de la ovodonación, analizando su tratamiento en la legislación peruana, en la doctrina nacional y en la jurisprudencia, con referencias al derecho comparado, incluyendo una revisión del tema aplicando la lógica, para emitir finalmente, desde una perspectiva jurídica, un juicio de valor sobre esta práctica que viene dándose de hecho en nuestro medio al amparo de una discutible interpretación legal.

\section{Palabras clave}

Ovodonación, donación de óvulos, cesión de óvulos, reproducción asistida, vientre de alquiler, fecundación heteróloga.

\begin{abstract}
The article addresses the controversial issue of egg donation, analyzing the way it is treated under Peruvian law, national doctrine, and jurisprudence, with references to comparative law, including a review of the subject applying logic to finally issue from a legal perspective a value judgment on this practice that has been actually taking place in our country under questionable legal interpretation.
\end{abstract}

\section{Key words}

Egg Donation, transfer of eggs, assisted reproduction, surrogacy, heterologous fertilization.

\footnotetext{
* Abogado y Magister en Derecho Civil y Comercial. Profesor de la Universidad Femenina del Sagrado Corazón, Universidad de Lima, ESAN y Universidad César Vallejo (Trujillo). Profesor de la Maestría en Bioética y Biojurídica de la Universidad Santo Toribio de Mogrovejo. Miembro de la Academia de Derecho y Ciencias Sociales de Córdoba. Ha sido Decano de la Facultad de Derecho de la UNIFE, Superintendente Nacional de la SUNARP, Presidente del Consejo del Notariado y Miembro del Comité de Bioética de la Facultad de Biología de la Universidad Nacional Mayor de San Marcos.
}

Fecha de recepción: 29/09/2014

Fecha de aceptación: 30/10/2014 


\section{Introducción}

El presente trabajo tiene por finalidad analizar un tema muy puntual y discutido: la viabilidad legal de la aplicación en nuestro país de la "cesión de óvulos", llamada también "donación de óvulos" u "ovodonación" 1 .

Vale decir que la importancia del contenido del debate bioético en general, el énfasis con el que se defienden determinadas posiciones extremas, así como la necesidad de preservar un sistema jurídico que repose en valores fundamentales, ponen de relieve la exigencia de buscar argumentos cada vez más sólidos y convincentes para la resolución de problemas bioéticos sustantivos.

En ese propósito, nos encontramos -siguiendo a Manuel Atienza-, con 3 formas de acercamiento o de concepciones de la argumentación ${ }^{2}$ : la formal, la material y la pragmática, las cuales pueden definirse como sigue:

a) El enfoque formal: Tiene un carácter técnico, pudiendo hacerse abstracción de la verdad o corrección de la premisa o conclusión, para fijarse en el procedimiento meramente formal y establecer determinados criterios de validez a partir de reglas de inferencia.

b) El enfoque material: Su preocupación esencial es más bien determinar la verdad de los enunciados, tratando de llegar a ella a través de diferentes medios racionalmente aceptables.

c) El enfoque pragmático: Ve la argumentación como un tipo de actividad lingüística, privilegiando no la dimensión sintáctica o semántica del lenguaje, sino la pragmática, vista como una actividad interactiva, buscando la persuasión.

El problema que se encuentra en esta tarea es que, en la discusión bioética, muchas veces solo se enfatiza uno u otro ámbito, ya sea por la insuficiencia de conocimientos, la falta de rigurosidad, porque nos dejamos llevar por los prejuicios o seducir por la retórica, prescindiendo de argumentos que puedan ser suficientemente convincentes por su rigor y carácter científico.

\footnotetext{
${ }^{1} \mathrm{Si}$ bien estos dos últimos términos resultan de por sí técnicamente cuestionables, ya que, para el Derecho Civil, la donación es un contrato $y$, por tanto, un negocio de naturaleza patrimonial -carácter completamente ajeno a la materia-, recurrimos a dichos términos únicamente teniendo en cuenta su uso común.

${ }^{2}$ Atienza, Manuel (2013). Curso de Argumentación Jurídica. Madrid: Trotta, p. 111.
} 


\section{El Problema}

Existen en nuestro país diversos institutos o clínicas de fertilización asistida que vienen aplicando la cesión de óvulos (como también la de espermatozoides, por supuesto), "servicio" que se publicita abiertamente, argumentando que ella está permitida en nuestra legislación. Resulta pertinente, por tanto, interrogarnos si ello es cierto.

Para contestar dicha pregunta, debemos partir del artículo $7^{\circ}$ de la Ley General de Salud (Ley 26842), el cual a la letra dice:

"Artículo $7^{\circ}$.- Toda persona tiene derecho a recurrir al tratamiento de su infertilidad, así como a procrear mediante el uso de técnicas de reproducción asistida, siempre que la condición de madre genética y de madre gestante recaiga sobre la misma persona.

Para la aplicación de técnicas de reproducción asistida, se requiere del consentimiento previo y por escrito de los padres biológicos.

Está prohibida la fecundación de óvulos humanos con fines distintos a la procreación, así como la clonación de seres humanos".

De acuerdo a dicha norma, entonces, se reconoce en primer término el derecho de toda persona de tratar su infertilidad y recurrir a las técnicas de reproducción asistida, "siempre que la condición de madre genética y de madre gestante recaiga sobre la misma persona".

El legislador, de esta manera, dispone con toda claridad que estas técnicas solo pueden aplicarse cuando quien aporte el óvulo y quien haga el embarazo sean la misma persona, con lo que, en cualquier caso en que dicha condición no se cumpla, sería inaplicable recurrir a estas técnicas.

Así, tanto en los casos del "alquiler de vientre", como en el caso de la "donación de óvulos", en los que madre genética y madre gestante no son la misma persona, no cabría apelar a tales técnicas. Sin embargo, hay quienes -incluyendo un fallo de la Corte Suprema- sostienen que la norma se refiere solo al primer caso, mas no al segundo. ${ }^{3}$

\footnotetext{
3 Así, por ejemplo: Mosquera Vásquez, Clara. "Las técnicas de procreación asistida en los tribunales peruanos". En: Revista Diálogo con la Jurisprudencia No. 147. Lima: Gaceta Jurídica, diciembre 2010, p. 149. Para una amplia aproximación, en cambio, a diversos cuestionamientos que plantean la fecundación artificial en general, léase: Morán de Vicenzi, Claudia (2008). "La Filiación y la Fecundación Artificial. En: Varios Autores. Temas de Bioética y Derecho. Lima: Facultad de Derecho de la Unifé - Cátedra Unesco de Bioética y Biojurídica. Por su parte, Carlos Fernández Sessarego, ponente del Libro I del Código Civil, señala que "Resultaría patética la situación de seres humanos que, al ser producidos en probeta en base a donantes anónimos, desconocen a sus progenitores y carecen de familia natural". Fernández Sessarego, Carlos (2012). Derecho de las Personas. XEdición. Lima: Grijley p. 54.
} 
¿Cuál es la interpretación correcta? ¿Puede aplicarse válidamente o no la "ovodonación" en el Perú? ¿Cuáles son los argumentos que se esgrimen para sostener que la norma bajo comentario solo se aplica para el caso del llamado "alquiler de vientre" y no para éste?

\section{El Primer Pronunciamiento de la Corte Suprema sobre la materia}

Para estudiar mejor el tema, pasemos a revisar el reciente primer caso en el que la CorteSuprema del Perú se pronunciara sobre la materia:

Se trata -en términos de breves- de una pareja que recurre a una clínica de fertilización porque tenía problemas para concebir, suscribiendo 3 documentos: Uno en el que declaran haber sido informados debidamente del proceso y del costo de la criopreservación de embriones; otro por el que el demandante acepta el almacenamiento de su semen por tiempo indeterminado; y uno tercero por el que declaran poseer buen estado psicológico y que el concebido tendrá la condición de hijo, asumiendo la pareja los deberes y derechos propios de la condición de padres; adicionalmente, renuncian a cualquier acción civil u otra con relación a lo establecido.

Es así que la pareja tuvo una niña. Sin embargo, poco después, ambos empezaron a tener problemas, derivando la situación en que el hombre solicite la nulidad del primer y tercer documento mencionado, invocando el art. V del Título Preliminar y el inc. 4) del artículo $219^{\circ}$ del Código Civil, argumentando que la niña había sido concebida usando los gametos de él, pero no los de ella, quien le había ocultado su infertilidad y había recurrido a una dadora anónima de óvulos.

La demanda es declarada infundada en todos sus extremos en primera instancia. Apelada la misma, la 6 a Sala Civil de la Corte Superior de Justicia de Lima, revocó la sentencia anterior y, reformándola, declaró fundada la demanda $y$, en consecuencia, nulos los actos jurídicos detallados en la misma. $^{4}$

\footnotetext{
${ }^{4}$ Mediante sentencia de fecha 31.08.2010, recaída en el expediente No. 1310-2010-Lima, la Sexta Sala Civil de la Corte Superior de Justicia de Lima resolvió la acción de nulidad de acto jurídico presentada por C.O.Q.C. contra M.A.D., declarando nulos los actos jurídicos de autorización de fertilización in vitro y transferencia embrionaria, al haberse usado un óvulo de una mujer distinta a la gestante.
} 
La Sala Suprema en lo Civil resolvió con fecha 11.08.2012 la casación interpuesta por M.A.A.D., declarando fundada la casación, nula la sentencia de vista y confirmada la sentencia de primera instancia, que declaró infundada la demanda (CASNo.4323-2010).

La Corte Suprema parte de señalar que el artículo $7^{\circ}$ de la Ley 26842 admite las técnicas de reproducción asistida como métodos supletorios no alternativos, agregando que la maternidad subrogada o vientre de alquiler no se encuentra reconocida legalmente en nuestro país ${ }^{5}$; empero, en el caso de la ovodonación, indica que si bien no se encuentra legislado, en virtud del axioma jurídico de que "todo lo que no está prohibido está permitido", el procedimiento de ovodonación "no es ilícito ni constituye delito, constituyendo más bien un vacío normativo y jurisprudencial". ${ }^{6}$

\section{Críticas al fallo de la Corte}

La sustentación que ofrece en este caso la Corte Suprema resulta discutible pues, con el mismo criterio, en una argumentación a pari, podría decirse entonces que como en nuestro país tampoco está legislado el vientre de alquiler y no está prohibido, entonces también podría aplicarse válidamente.

\footnotetext{
El demandante hab́a sustentado su pretensión en que dichos actos iban contra el art. V del Título Preliminar del Código Civil que sanciona con nulidad los actos que van contra el orden público y las buenas costumbres. Asimismo, invocó el art. 7 de la Ley General de Salud, argumentando que conforme al mismo, no es viable legalmente en el Perú la figura de la donación de óvulos.

La Corte consideró que "resulta evidente que los declarantes estaban autorizando la realización de un procedimiento contrario a una norma prohibitiva como el artículo 7 de la Ley General de Salud", declarando fundada la demanda y nulos los actos jurídicos de "Autorización de fertilización in vitro y Transferencia Embrionaria", asf como el "Convenio de Técnicas de Reproducción Asistida", materia del referido proceso.
}

Discrepando con el resto de Vocales, el magistrado Rivera Quispe observó que un acto jurídico nulo puede ser fuente de efectos, aún de aquellos negociales, en la medida que las partes, si bien no vinculadas, han ejecutado prestaciones; agrega que la nulidad de los actos cuestionados no presenta efecto jurídico que puede ser privado y/o asimismo es imposible volver a su estado anterior, y pretender desaparecer los derechos y obligaciones que hubieren nacido, advirtiendo además -entre otras consideraciones- que la pretendida nulidad, no puede afectar la eficacia del acto realizado. Con ello, plantea que se revoque la sentencia y reformándola se declare improcedente.

5 Sobre el tema, opina Junquera que “Creemos, pues, que lo más oportuno es no autorizar este tipo de sustitución maternal, puesto que los riesgos, peligros y problemas que ocasiona son demasiado elevados. Sin embargo, se podía (sic) autorizar por el juez, previo asesoramiento de la comisión nacional de reproducción asistida, en algún caso concreto, cuando se llevase a cabo en el entorno familiar, por motivos altruistas o de solidaridad con un miembro de la familia". Junquera de Estéfani, Rafael (1998). Reproducción Asistida, Filosofia Ética y Filosofía Jurídica. Madrid: Editorial Tecnos, p. 139.

${ }^{6}$ Es de señalar que, en favor de lo resuelto por la Corte, se ha señalado que las prohibiciones no pueden interpretarse de manera analógica, sino que deben ser explícitas; que lo que se plantea en el art. 7 es una exhortación y no una exigencia; que admitir la fecundación heteróloga solo con material genético masculino sería discriminador y que igual podrían haber conflictos de identidad en este último caso; agregándose además que la norma no impide la matemidad subrogada. Siverino, Paola. "Una mirada desde la bioética jurídica a las cuestiones legales sobre la infertilidad en el Perú". En: Revista Peruana de Ginecología y Obstetricia No 58. Lima: 2012, p. 216. 
Por otro lado, si además tenemos en cuenta la existencia del principio jurídico de que "no se puede distinguir donde la ley no distingue", no habría. que hacer diferencias entre uno y otro caso.

Más aún, si bien existe el principio de que "todo lo que no está prohibido está permitido", la Sala olvida que el derecho está compuesto por normas tanto escritas como no escritas, explícitas como implícitas, que hay prohibiciones expresas pero también tácitas. Si asumimos el criterio expuesto por la Corte, entonces habría que concluir que, como no está prohibido subastar un embrión, celebrar un contrato de donación con mi perro o que una persona se bañe en la pileta de la Plaza de Armas, cualquiera podría realizar dichos actos, lo cual sería absurdo.

Adicionalmente, estimamos que no se trata solo de una sugerencia, recomendación o invocación, pues mal podría figurar en esos términos en una ley, que es de carácter obligatorio y, más todavía, de carácter público.

Por último, consideramos que no hay discriminación alguna, pues la restricción en el caso de las mujeres obedece al hecho objetivo que son ellas quienes hacen el embarazo y, en quienes por tanto, puede surgir con mayor posibilidad el conflicto de encariñarse con el bebé que vienen gestando para otra.

\section{La "Ovodonación" en la Doctrina Nacional}

En la doctrina nacional, Cabrera Palomino, quien ha hecho un estudio específico sobre la donación de óvulos en el Perú, considera que: "Está demostrado que con la ejecución del procedimiento de ovodonación de las técnicas de reproducción asistida se violenta el derecho a la identidad del nacido, el mismo que debe sobreponerse aún sobre el derecho a la intimidad o privacidad delos padres" ${ }^{7}$

A su vez, Rodríguez-Cadilla, sostiene, invocando el bien del concebido, que: "Rechazamos la maternidad subrogada o maternidad por sustitución por considerarla inmoral, aunque no medie comercio y se haga por motivos

\footnotetext{
${ }^{7}$ Cabrera Palomino, Vanessa. Cabrera Palomino, Vanessa (2013). "La afectación del derecho de identidad de los nacidos bajo la ejecución del procedimiento de ovodonación por la inseguridad jurídica para la determinación de la filiación materna". En: Alétheia, Revista de la Escuela de Postgrado de la Universidad Femenina del Sagrado Corazón, año 1, No. 1, enero-diciembre 2013. Lima: UNIFE, p. 105.
} 
altruistas" ${ }^{8}$ En los mismos términos, se pronuncia Valverde Morante. ${ }^{9}$

Guevara Pezo, por su lado, considera que debe negarse expresamente la validez y licitud de los alquileres de vientre escondidos bajo el eufemismo de la expresión "madre sustituta". 10

Carlos Cárdenas Quirós, años antes, ya se había pronunciado tajantemente contra la fecundación heteróloga, considerando que "ningún ser humano tiene derecho, desde un punto de vista ético, a disponer de sus componentes genéticos" ${ }^{\mathbf{1 1}}$, participando enteramente de lo sostenido en la Instrucción vaticana sobre problemas de bioética respecto de la fecundación extracorpórea heteróloga. ${ }^{12}$

Comentando la casación referida, discrepa de la misma Analucía Torres, estimando que los casos de ovodonación se encuentran claramente prohibidos por el artículo $7^{\circ}$ de la Ley General de Salud, planteando incluso una interpretación que cuestionaría la aplicación de la fecundación asistida con intervención de un tercero dador de esperma. ${ }^{13}$

Esta última posición se contrapone a la de Juan Espinoza para quien, en vía de interpretación constitucional debe dejarse de lado el texto literal del artículo 7 de la Ley General de Salud y admitir la ovodonación, pues "prohibir la cesión de óvulos y no la de espermatozoides es evidentemente discriminatorio" ${ }^{14}$

Varsi, por su parte, luego de mencionar que en nuestro país está prohibida la ovodonación (fecundación extracorpórea con óvulo de cedente),

8 Rodríguez Cadilla Ponce, Rosario (1997). Derecho Genético: Técnicas de Reproducción Humana Asistida. Su trascendencia jurídica en el Perú. Lima: Editorial San Marcos, p. 244.

${ }^{9}$ Valverde Morante, Ricardo. Valverde Morante, Ricardo (2001). Derecho Genético. Reflexiones juridicas planteadas por las técnicas de reproducción humana asistida. Lima: Grafica Horizonte, p. 157.

10 Guevara Pezo, Víctor (2002). "Vacíos en el sistema legal en materia de biojurídica". En: Varios autores. Bioética y Biojurídica. La Unidad de la Vida. Lima: Ediciones Jurídicas UNIFE, p. 142.

${ }^{11}$ Cárdenas Quirós, Carlos (1994). Estudios de Derecho Privado (Reflexiones de un tiempo). Tomo I. Lima: Ediciones Jurídicas, p. 85 .

12 Ibidem, p. 86.

13 Torres Flor, Analucía. Derecho a la identidad y reproducción humana asistida heteróloga. Arequipa, Universidad Católica San Pablo, 2014, p. 86 y 91.

${ }^{14}$ Espinoza Espinoza, Juan (2012). Derecho de las Personas. Tomo I. Lima, Grijley, p. 152. 
la embriodonación (transferencia de embrión ajeno) y la maternidad portadora (servicios de gestación subrogada), manifiesta que "se trata de restricciones éticas al contrariar instituciones jurídicas como el parentesco, la filiación, el ejercicio natural del derecho reproductivo; pero, como actos médicos, carecen de sanción penal. En buena cuenta no son actos arreglados a Derecho, ni a la moral, pero al no estar tipificados en la ley penal, no son delitos ni falta". ${ }^{15}$

En términos generales, puede advertirse que existe una amplia opinión doctrinaria en el sentido de interpretar que el art. $7^{\circ}$ de la Ley General de Salud, es claro al prohibir la ovodonación. Este criterio comparten, aparte de los autores mencionados, Luis Cárdenas Rodríguez ${ }^{\mathbf{1 6}}$ Alberto González Cáceres ${ }^{17}$, Jairo Cieza Moza ${ }^{18}$ y Claudia Morán. ${ }^{19}$ A favor de la validez de la interpretación de la Corte, se pronuncia Mosquera Vásquez. ${ }^{20}$

\footnotetext{
${ }^{15}$ Varsi, Enrique (2013). Derecho Genético. Principios generales. $5^{a}$ edición. Lima: Grijley, .p. 434. Empero, observa el mismo autor que la ley peruana no se ha pronunciado sobre el caso de la mujer que acepta ser inseminada con el semen del marido de otra a fin de entregar a la criatura una vez nacida, caso entonces en el que coincide la madre genética y la madre gestante. Este caso no estaría prohibido por la norma de salud, por lo que tendría que resolverse al amparo del artículo $409^{\circ}$ del Código Civil que establece que la maternidad se define por el parto. Adicionalmente, podría invocarse la norma $\mathrm{V}$ del Título Preliminar del Código Civil que sancionan los actos que van contra el orden público y las buenas costumbres.
}

\section{6} 16 "Con facilidad se entiende, entonces, que si la ovodonación implica una disociación entre maternidad genética y maternidad gestacional, al recaer dichas calidades en personas distintas, se encuentra incluida dentro de la previsión normativa, la cual establece como una necesidad el que ambos tipos de maternidad recaigan en una sola persona.

De modo que no se encuentra explicación para la decisión de la Sala Suprema de entender que la ovodonación está permitida por existir un vacío legal". Cárclenas Rodríguez, Luis. "Maternidad por ovodonación". En: Revista Diálogo con la Jurispudencia No. 166. Lima: Gaceta Juridica, julio 2012, p. 72.

17 González Cáceres, Alberto. "Cuando mi madre es un número. Identidad genética e interés superior del niño". En: Revista Juridica del perú. No. 93. Lima: noviembre 2008, p. 6. E1 artículo puede encontrarse en: http://www.sodeme.org/publicaciones/articulos/a_01_15.pdf, p. 6.

\footnotetext{
18

Como bien señala Cieza, sin perjuicio de que la ovodonación esté prohibida, no cabe duda que el hecho concreto es que, en el caso comentado, hay una niña que ha sido reconocida por la madre gestante (la demandada) y en aras del beneficio de la menor reconocida debe mantenerse su situación jurídica para no dejarla en la incertidurmbre, más aún cuando la donante del ovulo es anónima; ello sin perjuicio de que la menor, cuando tenga discernimiento, pueda conocer su origen biológico sin que ello genere vínculos filiatorios. Cieza Mora, Jairo. "La fécnica de reproducción humana asistida y la Corte Suprema. ¿Quién es la madre?" En: Revista Diálogo con la Jurisprudencia No.121. Lima: octubre 2008, p. 27.

19

Morán de Vicenzi, Claudia (2008). Op. cit., p. 159

20 Mosquera Vásquez, Clara Celinda. "El primer caso de ovodonación e la Corte Suprema". En: Revista Diálogo con la Jurisprudencia No. 166. Lima: Gaceta Jurídica, julio 2012, p.70.
} 
Finalmente, parece interesante la hipótesis que plantea Cárdenas Rodríguez en el sentido que la Corte, al aceptar la ovodonación, lo habría hecho para pretender evitar la nulidad establecida en el art. V del Título Preliminar del Código Civil y no afectar la maternidad legal de la cedente del óvulo respecto a la niña; empero, coincidimos en que la maternidad legal queda fuera de los efectos de la nulidad, pues dado el hecho del nacimiento de la menor y si la situación surge la necesidad de determinar quién es la madre, por más que la ovodonación se encuentre prohibida, se trata de cosas que merecen tratarse jurídicamente en forma independiente.

\section{Referencias en la Legislación Comparada}

Vale señalar que, en Austria, al estar prohibida la donación de óvulos y esperma, el caso fue llevado ante el Tribunal Europeo de Derechos Humanos, el cual declaró la validez de la prohibición austriaca, al considerar que dicha restricción no viola la Convención Europea de Derechos Humanos.

Asimismo, cabe mencionar que la donación de óvulos está prohibida en Alemania, Croacia, Italia, Lituania, Noruega y Turquía (incluso la donación de esperma está prohibida en Italia, Lituania y Turquía), entre otros países.

En Suiza, el literal d) del artículo $119^{\circ}$ de la Constitución, prohíbe expresamente todas las formas de maternidad de sustitución.

\section{Un Planteamiento Lógico-formal de la materia}

El tema también puede ser analizado desde un punto de vista lógico formal, partiendo de que tenemos que el enunciado general del art. $7^{\circ}$ de la Ley de Salud plantea lo siguiente:

" $\mathrm{p}$ " = La madre genética y la madre gestante son la misma persona.

" $\mathrm{q}$ " = Se puede recurrir válidamente a las técnicas de reproducción asistida.

Luego: $\mathrm{p} \leftrightarrow \mathrm{q}(1)$

Ahora bien, podemos mencionar como casos en los que la madre genética y madre gestante no son la misma persona, los siguientes: 
" $\mathrm{r}$ " = "alquiler de vientre"

"s" = "donación de óvulos".

Luego tenemosentonces: ( $r \quad v \quad s) \rightarrow \sim p$

De (1) y (2), se deduce entonces lo siguiente ${ }^{21}$.

$(p \mid \leftrightarrow q) \mid \wedge((r|\vee| s)[\rightarrow \mid \sim p): \Rightarrow:(r \mid \rightarrow \sim q) \vee(s \mid \rightarrow \sim q)$

Seguidamente, hacemos el desarrollo respectivo de las Tablas de Verdad, de lo que se deriva lo siguiente:

\begin{tabular}{|c|c|c|c|c|c|c|c|c|c|c|c|c|c|c|c|c|}
\hline & & $q$ & & & & & & & & $(1$ & & $\sim q$ & $V$ & & & \\
\hline & $\mathbf{V}$ & $V$ & $\mathbf{F}$ & $V$ & V & $y$ & $F$ & $\mathbf{F}$ & $V$ & $\mathrm{~V}$ & $F$ & $\mathrm{~F}$ & $F$ & & $F$ & \\
\hline & $\mathbf{V}$ & $V$ & $F$ & $\mathrm{~V}$ & $\mathbf{V}$ & $F$ & $F$ & $\mathbf{F}$ & $\mathrm{V}$ & $V$ & $\mathbf{F}$ & $F$ & $\mathbf{V}$ & $=$ & $V$ & \\
\hline & $V$ & $V$ & $F$ & $F$ & $\mathbf{V}$ & $V$ & $F$ & & $V$ & $F$ & $V$ & $F$ & $\mathbf{V}$ & V & & \\
\hline & $\mathbf{V}$ & $V$ & $\mathbf{V}$ & $F$ & $F$ & F & $\mathbf{v}$ & & $V$ & $F$ & v & $F$ & $v$ & & $\mathbf{v}$ & \\
\hline 1 & $\mathbf{F}$ & $F$ & $\mathbf{F}$ & V & V & $V$ & $\mathbf{F}$ & $\mathbf{F}$ & V & $V$ & V & $V$ & $\mathbf{V}$ & $V$ & $\mathbf{V}$ & \\
\hline$y$ & $\mathbf{F}$ & 5 & $F$ & $V$ & $\mathbf{V}$ & $F$ & $F$ & & $V$ & $V$ & $\mathbf{V}$ & $\mathrm{V}$ & $\mathbf{V}$ & $F$ & $\mathbf{V}$ & \\
\hline 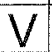 & $\mathbf{F}$ & $t$ & $\mathbf{r}$ & $F$ & V & & $\mathbf{F}$ & & $V$ & $F$ & $v$ & $V$ & & 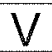 & $\mathbf{V}$ & \\
\hline I & $F$ & $\Gamma$ & $\mathbf{V}$ & $F$ & $F$ & & $\mathbf{V}$ & $F$ & $\mathrm{~V}$ & $F$ & $\mathbf{V}$ & $\mathrm{V}$ & $\mathbf{V}$ & $F$ & $\mathbf{V}$ & \\
\hline$E$ & $F$ & $V$ & $\mathbf{F}$ & $\mathrm{V}$ & $\mathbf{V}$ & 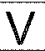 & $\mathbf{V}$ & $V$ & V & $\mathrm{V}$ & $F$ & $F$ & $F$ & $V$ & $\mathbf{F}$ & \\
\hline[ & $F$ & $V$ & $\mathbf{F}$ & $V$ & $\mathbf{V}$ & & $\mathbf{V}$ & & $V$ & $V$ & & $F$ & & $F$ & $\mathbf{V}$ & \\
\hline & $\mathbf{F}$ & $\mathrm{V}$ & $F$ & $F$ & $\bar{V}$ & & $\mathbf{V}$ & V & V & $\mathrm{F}$ & $\mathbf{V}$ & $F$ & $\mathbf{V}$ & $V$ & $\mathbf{F}$ & \\
\hline & $\mathbf{F}$ & $V$ & $\mathbf{F}$ & $F$ & $F$ & & $\mathbf{V}$ & & $V$ & $F$ & $V$ & $F$ & V & $F$ & $\mathbf{V}$ & \\
\hline & $\mathbf{V}$ & 5 & $V$ & $V$ & $V$ & & $\mathbf{V}$ & & $V$ & $V$ & $\mathbf{V}$ & $\mathrm{V}$ & 1 & $V$ & $\mathbf{V}$ & \\
\hline & $\mathbf{V}$ & & & $\bar{V}$ & $\mathbf{V}$ & & $\mathbf{V}$ & & $V$ & $V$ & $\mathbf{V}$ & V & V & $F$ & $\mathbf{V}$ & \\
\hline & $\mathbf{V}$ & & $\mathbf{V}$ & $F$ & $\mathbf{V}$ & & $\mathbf{V}$ & & $V$ & & $\mathbf{V}$ & $V$ & 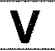 & $/$ & $\mathbf{V}$ & \\
\hline & $\bar{V}$ & & $\mathbf{V}$ & $\mathrm{F}$ & $\mathbf{F}$ & & V & & $V$ & & & $V$ & & & & \\
\hline
\end{tabular}

Por tanto, en estricta lógica, ni en el caso de "alquiler de vientre" ni en el caso de "ovodonación", procede válidamente recurrir a las técnicas de reproducción asistida.

21 Dicho en términos lingüísticos: En tanto la madre genética y la madre gestante sean la misma persona, entonces es válido recurrir a las técnicas de reproducción asistida. Mas resulta que en las figuras del "alquiler" de vientre o la "donación" deóvulos, no se da que la madre genética y la gestante sean la misma persona. Entonces ni es válido el alquiler de vientre, ni es válida la donación de óvulos. 


\section{Conclusiones}

1. Consideramos que la Ley General de Salud es clara al admitir, a través de su artículo 7, el uso de las técnicas de reproducción asistida solo cuando la madre genética y la madre gestante sean la misma persona. En tal virtud, la ovodonación, en estricto derecho, resulta siendo ilegal.

2. Sin perjuicio de ello, resulta claro que parece mucho más reprochable el "alquiler de vientre" que la "ovodonación", como también es mucho más reprochable la venta de óvulos que una cesión por motivos meramente altruistas.

3. Es entendible también el justo deseo de una mujer de poder tener hijos recurriendo a esta técnica, en caso de no haber podido optar exitosamente por otras alternativas, mas, para ello, estimamos que se requeriría, en todo caso, que se modifique la ley y no forzar interpretaciones ajenas al texto y al espíritu mismo de la norma vigente. Un deseo de por sí, no constituye un derecho.

4. Es importante promover una mayor aplicación de la Lógica tanto en el análisis jurídico como en el bioético, pues puede servir para facilitar un mejor enfoque argumentativo en la sustentación de casos, ofreciendo nuevos recursos para la discusión racional y la difusión del conocimiento. ${ }^{22}$

5. Resulta necesario incidir en el estudio de la Bioética tanto en los magistrados como en los operadores y estudiantes de derecho en general, teniendo en cuenta la delicada naturaleza de las cuestiones que deben y deberán abordar, en forma cada vez más exigente, vinculadas con aspectos fundamentales de nuestra existencia humana.

En tal sentido, resultan sumamente significativas para concluir este estudio, las siguientes palabras del profesor español José López Guzmán: "La clara conciencia de la importancia actual de la Bioética ha impulsado su progresiva implantación en el panorama educativo universitario. No obstante, su incorporación a los diferentes niveles de enseñanza ha sido más

\footnotetext{
22 "Pero también los que no se dedican a las matemáticas harán bien en estudiar al menos en sus rasgos fundamentales las leyes de la lógica, que nos incitan a observar una especie de "higiene mental" y nos obligan a formular limpiamente nuestras ideas y pensamientos". Drosser, Christoph (2013). La seducción dela lógica. Barcelona: Ariel, p.10.
} 
lenta de lo que cabría esperar, fundamentalmente en la formación de pregrado y de grado. Ello se debe a que, como es bien conocido, la cultural actual es tremendamente pragmática y utilitarista, y en un mundo que da primacía a lo demostrable y ponderable, la aportación de la Bioética es difícilmente cuantificable ${ }^{\prime \prime}{ }^{23}$

\section{Referencias}

- Atienza, Manuel. (2013) Curso de Argumentación Jurídica. Madrid: Trotta.

- Cabrera Palomino, Vanessa. (2013) "La afectación del derecho de identidad de los nacidos bajo la ejecución del procedimiento de ovodonación por la inseguridad jurídica para la determinación de la filiación materna". En: Alétheia, Revista de la Escuela de Postgrado de la Universidad Femenina del Sagrado Corazón, año 1, No. 1, enero diciembre 2013. Lima: UNIFÉ.

- Cárdenas Quirós, Carlos. (1994) Estudios de Derecho Privado (Reflexiones de un tiempo). Tomo I. Lima: Ediciones Jurídicas.

- Cárdenas Rodríguez, Luis. (2012) "Maternidad por ovodonación". En: Revista Diálogo con la Jurispudencia No. 166. Lima: Gaceta Jurídica, Julio.

- Cieza Mora, Jairo. (2008) "La técnica de reproducción humana asistida y la Corte Suprema. ¿Quién es la madre? En: Revista Diálogo con la Jurisprudencia No. 121. Lima: octubre.

- Drosser, Christoph. (2013) La seducción de la lógica. Barcelona: Ariel.

- Espinoza Espinoza, Juan. (2012) Derecho de las Personas. Tomo I. Lima, Grijley,

- Fernández Sessarego, Carlos. (2012) Derecho de las Personas. X Edición. Lima: Grijley.

\footnotetext{
23 López Guzmán, José (2013). “La bioética personalista en los planes de estudio universitarios. Ens: Cuadernos de Bioética 2013/1" pp. 79-80.
} 
- González Cáceres, Alberto. (2008) “Cuando mi madre es un número. Identidad genética e interés superior del niño". En: Revista Jurídica del Perú. No. 93. Lima: noviembre.

- Junquera de Estéfani, Rafael. (1998) Reproducción Asistida, Filosofía Ética y Filosofía Jurídica. Madrid:Editorial Tecnos.

- López Guzmán, José. (2013) “La bioética personalista en los planes de estudio universitarios". En: Cuadernos de Bioética 2013/1 ${ }^{\text {a }}$.

- Morán de Vicenzi, Claudia. (2008) "La Filiación y la Fecundación Artificial". En: Varios Autores. Temas de Bioética y Derecho. Lima: Facultad de Derecho de la UNIFÉ - Cátedra Unesco de Bioética y Biojurídica.

- Mosquera Vásquez, Clara Celinda. (2012) „El primer caso de ovodonación en la Corte Suprema". En: Revista Diálogo con la Jurisprudencia No. 166. Lima: Gaceta Jurídica, julio.

- Mosquera Vásquez, Clara. (2010) "Las técnicas de procreación asistida en los tribunales peruanos". En: Revista Diálogo con la Jurisprudencia No. 147. Lima: Gaceta Jurídica, diciembre.

- Rodriguez-Cadilla Ponce, Rosario. (1997) Derecho Genético: Técnicas de Reproducción Humana Asistida, Su trascendencia jurídica en el Perú. Lima: Editorial San Marcos.

- Siverino, Paola. (2012) "Una mirada desde la bioética jurídica a las cuestiones legales sobre la infertilidad en el Perú". En: Revista Peruana de Ginecología y Obstetricia No 58. Lima.

- Torres Flor, Analucía. (2014) Derecho a la identidad y reproducción humana asistidaheteróloga. Arequipa, Universidad Católica San Pablo.

- Valverde Morante, Ricardo. (2001) Derecho Genético. Reflexiones jurídicas planteadas por las técnicas de reproducción humana asistida. Lima: Gráfica Horizonte.

- Varsi, Enrique. (2013) Derecho Genético. Principios generales. $5^{\mathrm{a}}$ edición. Lima: Grijley. 
